\title{
Electrokinetic motion of single nanoparticles in single PDMS nanochannels
}

\author{
Ran Peng and Dongqing Li* \\ Department of Mechanical and Mechatronics Engineering \\ University of Waterloo \\ Waterloo, Ontario, Canada N2L 3G1
}

*Corresponding author, Email: dongqing@uwaterloo.ca

The final publication is available at Springer via http://dx.doi.org/10.1007/s10404-017-1848-0 


\begin{abstract}
Electrokinetic motion of single nanoparticles in single nanochannels was studied systematically by image tracking method. A novel method to fabricate PDMS-glass micronanochannel chips with single nanochannels was presented. The effects of ionic concentration of the buffer solution, particle-to-channel size ratio and electric field on the electrokinetic velocity of fluorescent nanoparticles were studied. The experimental results show that the apparent velocity of nanoparticles in single nanochannels increases with the ionic concentration when the ionic concentration is low and decreases with the ionic concentration when the concentration is high. The apparent velocity decreases with the particle-to-channel size ratio $(\mathrm{a} / \mathrm{b})$. Under the condition of low electric fields, nanoparticles can hardly move in single nanochannels with a large particle-to-channel size ratio. Generally, the apparent velocity increases with the applied electric field linearly. The experimental study presented in this article is valuable for future research and applications of transport and manipulation of nanoparticles in nanofluidic devices, such as separation of charged nanoparticles and DNA molecules.
\end{abstract}

Keywords: Electrokinetic motion, Nanoparticles, PDMS nanochannels, Particle-tochannel size ratio 


\section{Introduction}

The phenomenon of electrophoresis describes the motion of charged colloidal particles suspended in electrolyte solutions in response to external applied electric fields (Li 2004; Masliyah and Bhattacharjee 2006) has long been applied to separate a mixture of charged particles or molecules practically by using porous media, such as gels (Unlu et al. 1997). The development of nanofabrication techniques today (Mijatovic et al. 2005; Xia et al. 2012; Duan et al. 2013) makes manipulation and transport of target molecules and particles in nanofluidic platforms possible. For example, stretching, separation and direct observation of DNAs (Napoli et al. 2010; Menard and Ramsey 2013; Yasui et al. 2013; Sackmann et al. 2014; Jeffet et al. 2016; Plesa et al. 2016) and proteins (Wang et al. 2005; Fu et al. 2007), sizing and detection of nanoparticles (Zhou et al. 2011; Davenport et al. 2012; Harms et al. 2015) by electrokinetically driven force in nanofluidics devices have been reported recently. However, to make full use of these techniques with high performance and efficiency, it is crucial to fully understand the mechanism of electrokinetic motion of nanoparticles or molecules in the nanoscale spaces, such as in straight nanochannels.

A large number of numerical and analytical models have been developed to study the electrophoresis behavior since the last century, and these models are based on different limiting assumptions and working conditions, such as domain boundary, particle shape and surface zeta potential, type of electrolyte solution, and thickness of electric double layer (EDL). In the early years, Helmholtz-Smoluchowski and Debye-Huckel models for electrophoresis of particles in open boundaries assume particle motion in stationary liquids under the conditions of thin $\operatorname{EDL}(k a \gg 1)$ and thick EDL $(k a \ll 1)$, respectively (Dukhin 1993; Delgado et al. 2007), where $a$ is the radius of the particle, and $k^{-1}$ is the Debye length. Two general formulas for the electrophoretic mobility of charged particles in concentrated electrolyte solutions and charged particles in extremely diluted electrolyte solutions were derived (Masliyah and Bhattacharjee 
2006). These two formulas are bridged by Henry's function valid for arbitrary $k a$ (Henry 1931) and further improved by Ohshima et al (Ohshima 1994; Ohshima 1996; Swan and Furst 2012). Modern theories focusing on the other working parameters and conditions have also been developed. These conditions can be divided into two branches, including the above-mentioned unbounded environment (Wiersema et al. 1966; Ohshima et al. 1983; Chen and Keh 1988) and the bonded domain which investigates the channel wall effects on the electrophoretic motion of particles. Particle motion near a flat plane (Keh and Anderson 1985; Keh and Chen 1988; Shugai and Carnie 1999), in rectangular channels (Movahed and Li 2012; Liu et al. 2016), cylindrical channels (Keh and Chiou 1996; Hsu and Chen 2007) with the conditions of thick EDL limit (Ennis and Anderson 1997; Hsu et al. 2004; Miloh and Boymelgreen 2014), thin EDL limit (O'Brien and Hunter 1981; Keh and Anderson 1985; Keh and Chen 1988; Hsu et al. 2012a) and arbitrary EDL thickness (Keh and Hsieh 2008; Chen and Keh 2013; Liu et al. 2014) has been studied. The thickness of EDL is dominated by the ionic concentration of the buffer solutions (Li 2004), however, pH values (Hsu et al. 2012b; Wang et al. 2013) and symmetry or asymmetry property of the ions (O'Brien and White 1978; Semenov et al. 2013; Nedelcu and Sommer 2014; Liu et al. 2016) also affect the electrophoretic behavior. For the cases of particle motion in cylindrical channels, generally, the particles move along the centerlines of the channels (Keh and Chiou 1996; Shugai and Carnie 1999; Tseng et al. 2013), however, the off-centerline effect has also been investigated (Zhang et al. 2009; Lee and Keh 2014; Liu et al. 2014). Zeta potential of the particle surfaces is another key parameter in the electrophoresis. Besides the general low zeta potential assumption (Ennis and Anderson 1997; Shugai and Carnie 1999; Hsu et al. 2004), particles with high zeta potential (Wiersema et al. 1966; O’Brien and White 1978; Liu et al. 2016), arbitrary zeta potential (Keh and Hsieh 2008) and particles with non-uniform zeta potential distribution (Qian et al. 2008; Wang and Keh 2009) have also been studied. In addition, electrophoresis models involving neutral particles and dielectric 
particles (Shugai and Carnie 1999; Miloh and Boymelgreen 2014) and particles made of soft materials (H. 1995; Ohshima 1995; Hsu et al. 2012a; Chen and Keh 2013; Tseng et al. 2013), porous media (Huang et al. 2012; Li and Keh 2016) as well as air bubbles (Schnitzer et al. 2014) have been investigated. It is also a fact that in the practical applications, the shape of the particles is diverse. As a result, modeling of electrophoresis of spherical (Wiersema et al. 1966; Keh and Anderson 1985; Keh and Chiou 1996; Ennis and Anderson 1997; Shugai and Carnie 1999; Miloh and Boymelgreen 2014; Liu et al. 2016), cylindrical (Hsu and Ku 2005; Hsu et al. 2012a), rectangular (Li and Daghighi 2010; Movahed and Li 2012) or spheroidal (Yoon and Kim 1989) particles has been conducted.

Experimental studies of electrokinetic motion of single particles provide fundamental understanding of electrokinetic transport phenomena. For example, the channel wall effects on the electrokinetic motion of individual spherical microbeads in PDMS microchannels have been studied by Xuan et al. (Xuan et al. 2005; Xuan et al. 2006). For transport of single nanoparticles in nanochannels, several numerical models (Liu et al. 2014; Hulings et al. 2015) were presented recently; however, systematic studies of electrokinetic motion of nanoparticles in nanopores or in straight nanochannels by experiments are very limited (Napoli et al. 2011; Han et al. 2012; Harms et al. 2015; Weerakoon-Ratnayake et al. 2016). One possible reason is that the nanochannels used in these studies are made of silicon, glass (Napoli et al. 2011; Harms et al. 2015) or thermal plastics (Weerakoon-Ratnayake et al. 2016), and high-cost, sophisticated equipment and complicated procedures are required for the device fabrication. In addition, the nanochannels used in these works are nano-slits that have only one dimension in the nanometer scale, while other dimensions of these nano-slits are larger than $5 \mu m$ (Napoli et al. 2011; Weerakoon-Ratnayake et al. 2016). Obviously, in these nano-slits, the nanoscale channel wall can influence the nanoparticle motion in one dimension. Furthermore, because of the micron size in other dimensions, such a nano-slit allows transport of multiple nanoparticles through 
the same cross-section of the channel simultaneously, and the interactions between the nanoparticles may affect the electrophoretic mobility. (Keh and Yang 1991; Keh and Chen 1993; Loewenberg and Davis 1995; Shugai et al. 1997; Ding and Keh 2001) Also, the ionic concentration of the buffer solutions is limited in a small range in these works, which is not sufficient to study the ionic concentration effects on the electrokinetic motion of nanoparticles. Electrophoresis of spherical nanoparticles in nanochannels with particle-to-nanochannel size ratio larger than 0.67 (Weerakoon-Ratnayake et al. 2016) is still a mystery.

This work investigates electrokinetic motion of single polystyrene nanoparticles in single PDMS nanochannels systematically. A novel method to fabricate PDMS-glass micronanochannel chips with single nanochannels smaller than $70 \mathrm{~nm}$ deep is presented. All the nanochannels used in this study are in nanoscale in both the width and the depth dimensions. Electrokinetic motion of single fluorescent nanoparticles in $\mathrm{LiBr}$ aqueous solutions from $10^{-5} \mathrm{M}$ to $10^{-2} \mathrm{M}$ through single nanochannels is measured by a fluorescent particle tracking system. Concentration effects, particle size to channel size ratio effects, and electric field effects on the electrokinetic motion of single nanoparticles are investigated quantitatively. An extreme case of large size ratio (as large as 0.9 ) is also examined.

\section{Experimental setup}

\subsection{Fabricating of micro-nanochannel chips}

Reliable and reproducible micro-nanofluidic chips are essential to the experimental studies of electrokinetic motion of nanoparticles in nanochannels. The procedures for fabricating micronanochannel chips are as the following. Firstly, a nanocrack with desirable location and size is generated on a polystyrene surface by solvent-induced method (Peng and Li 2015; Peng and Li 2016a). An artificial defect marked on the polystyrene surface is used to control the location of the nanocrack, and the location of the defect is controlled by the manipulation stage of a 
micro-hardness testing system (Peng and Li 2016a), and the size of the nanocrack is adjusted by controlling the working parameters of the solvent-induced method, i.e., the volume of the reagent, the heating time, the heating temperature and so on (Peng and Li 2015). Then, the negative nanocrack is replicated onto a piece of polymer slab (Smooth cast) surface by applying the nanoimprint method, and the optimal working parameters for this process can be found in the reference (Peng and Li 2016a). In the third step, a piece of PDMS slab with microchannel system composed of one pair of U-shaped negative microchannels is attached to another layer of flat PDMS slab. The PDMS slab with the microchannel system is replicated from a microchannel mold which is fabricated by the standard photolithography technique. The hollow microchannels are filled with liquid-form pre-curing smooth cast first. Then the flat PDMS slab is peeled off after 30 mins, leaving the rubber-like smooth cast inside the microchannels. After that, the PDMS slab with microchannels is attached to the plasma-treated smooth cast nanochannel mold that is positioned to bridge the two U-shaped microchannels. The smooth cast microchannel will be bonded onto the nanochannel mold surface due to the plasma treatment. Finally, a smooth cast micro-nanochannel mold is obtained by peeling off the PDMS microchannel. The smooth cast micro-nanofluidic channel mold is used to replicate the network of two U-shaped microchannels bridged by a nanochannel in a PDMS chip by applying the bi-layer strategy, with one thin layer of x-PDMS supported by a thick layer of regular PDMS (Peng and Li 2016a). Finally, a nanofluidic chip is obtained by bonding the PDMS micro-nanochannel chip onto a piece of glass slide after plasma treatment. Fig. 1 (a) shows an example of the micro-nanochannel chips and Fig. 1 (b) is a zoomed-in view of the nanochannel section. Fig. 1 (c) and Fig. 1 (d) are a 3D image of the nanochannel and three cross-sections of this nanochannel measured by an AFM (Multimode ${ }^{\mathrm{TM}}$ SPM, Digital Instruments), respectively. 


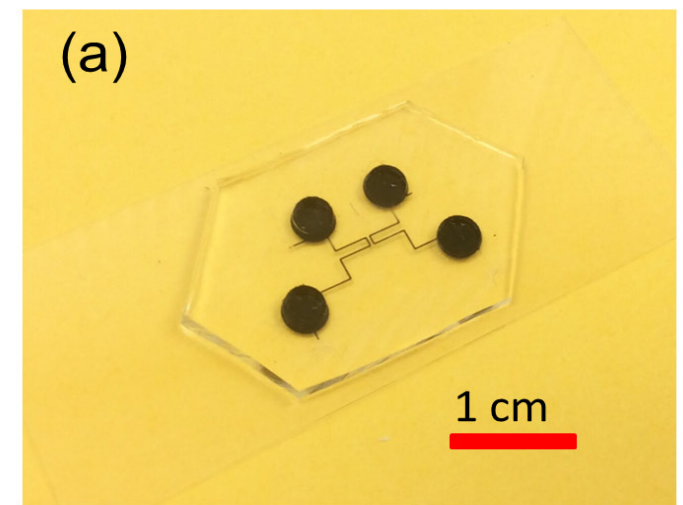

(c)

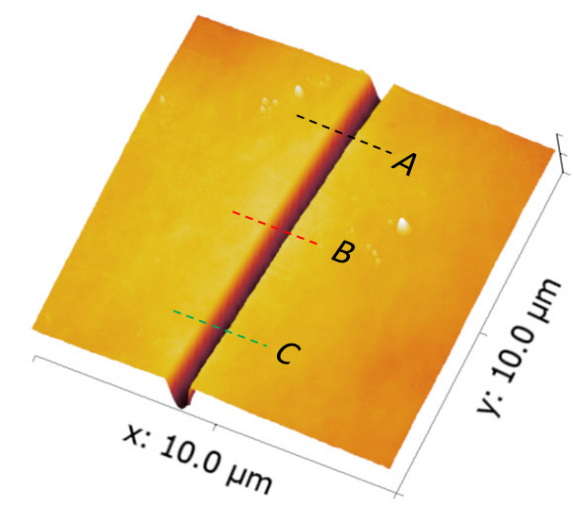

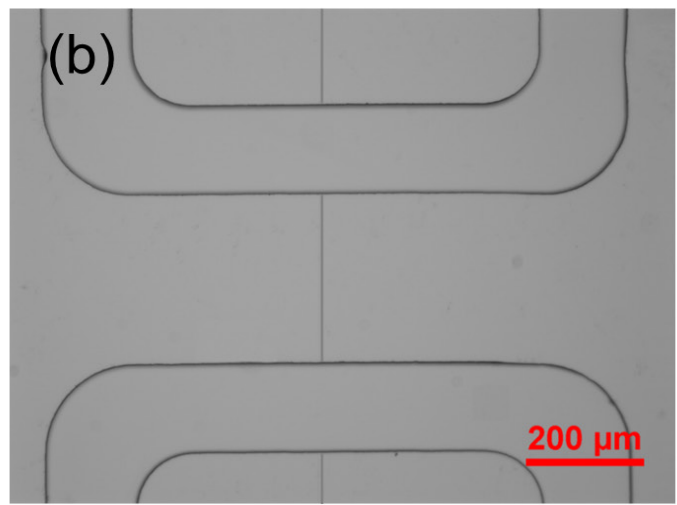

(d)

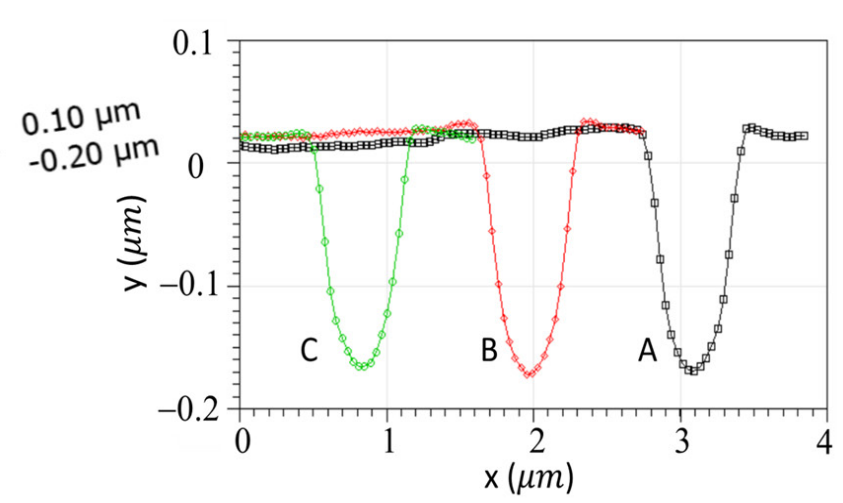

Fig. 1 (a) An example of the PDMS-glass micro-nanochannel chips, (b) a zoomed-in view of the micro-nanochannel conjunctions of the micro-nanochannel chip, (c) a 3D image of the nanochannel and (d) three cross-sections A, B and C of this nanochannel, separated by $3 \mu \mathrm{m}$ measured by the AFM. The nanochannel is about $610 \mathrm{~nm}$ wide and $180 \mathrm{~nm}$ deep.

\subsection{Fluorescent nanoparticle tracking system}

One direct way to determine the velocity of a fluorescent nanoparticle is to track the motion of the fluorescent nanoparticle by using a fluorescent microscope. Fig. 2 (a) shows a schematic diagram of the nanoparticle tracking system used in this study. This system is composed of a fluorescent microscope (Nikon Ti-E), a PDMS-glass micro-nanofluidic chip, a DC power source and a computer with image processing software (NIS-Elements BR). The fluorescent microscope is equipped with a $100 \times$ oil immersion objective lens (Apo $\lambda, N A=1.45$ ), a blue laser source $(\lambda=488 \mathrm{~nm})$ and a CCD camera (Q-imaging, Retiga 2000R). During the 
experiments, the micro-nanochannel chip is located on the working stage of the microscope with the nanochannel section on the focal point of the oil lens. Nanoparticle samples are loaded into the microchannels and the nanochannel through the channel wells. A DC power source (Keithley, 6517A) is employed to apply electrical field along the single nanochannel through two Platinum electrodes inserted in the wells at the end of the microchannels. In this way, nanoparticles are loaded into the nanochannel by electroosmotic flow. As the nanoparticles move in the nanochannels under the electric fields, the CCD camera will record the motion of the nanoparticles and the software will calculate the apparent velocity of the particles. Fig. 2 (b) shows an example of the trajectory of a $140 \mathrm{~nm}$ particle in a $340 \mathrm{~nm}$ deep nanochannel under an electric field of $2 \mathrm{kV} / \mathrm{m}$. The nanoparticle is in $10^{-4} \mathrm{M} \mathrm{LiBr}$ solution, and the average apparent velocity of this nanoparticle is $68.5 \mu \mathrm{m} / \mathrm{s}$. A video of the particle motion can be found in the Electronic Supplementary Material.

In this work, in order to study the electrokinetic motion of single nanoparticles in single nanochannels, only one single nanoparticle was introduced into the nanochannel each time. However, for most cases, both the PDMS nanochannel wall and the nanoparticles are negatively charged. As a result, the nanoparticles can hardly flow into the nanochannel freely even an external electric field (for example, $2 \mathrm{kV} / \mathrm{m}$ in the experiments) was applied along the nanochannel due to electrostatic repulsion between the channel wall and the nanoparticles. However, nanoparticle will accumulate at the entrance of the nanochannel due to the electrostatic attraction force. To "push" the nanoparticles into the nanochannel, a very strong electric field pulse (duration of less than $0.5 \mathrm{~s}$ ) was applied to the nanochannel; consequently, one or more nanoparticles were introduced into the nanochannel in a very short time due to the strong electrostatic force along the axis of the nanochannel. In the case of more than one nanoparticles were loaded into the nanochannel, a weak electric field was applied on the nanochannel to release some of the particles, finally leaving only one inside the channel. It 
should be noted that, because the nanochannels are in nanoscale in both the depth and the width directions, the nanoparticles inside the nanochannel will line up automatically due to the abovementioned electrostatic repulsion force between the channel wall and the nanoparticles. As a result, the releasing process can be controlled precisely. As only one nanoparticle was introduced into the channel, we switched the direction of the electric field of specific value back and forth to drive or manipulate the single nanoparticle and to measure the electrokinetic velocity, generally, for 10 time or more. After that, this particle was released, and another one was loaded, and so on and so forth. 
(a)

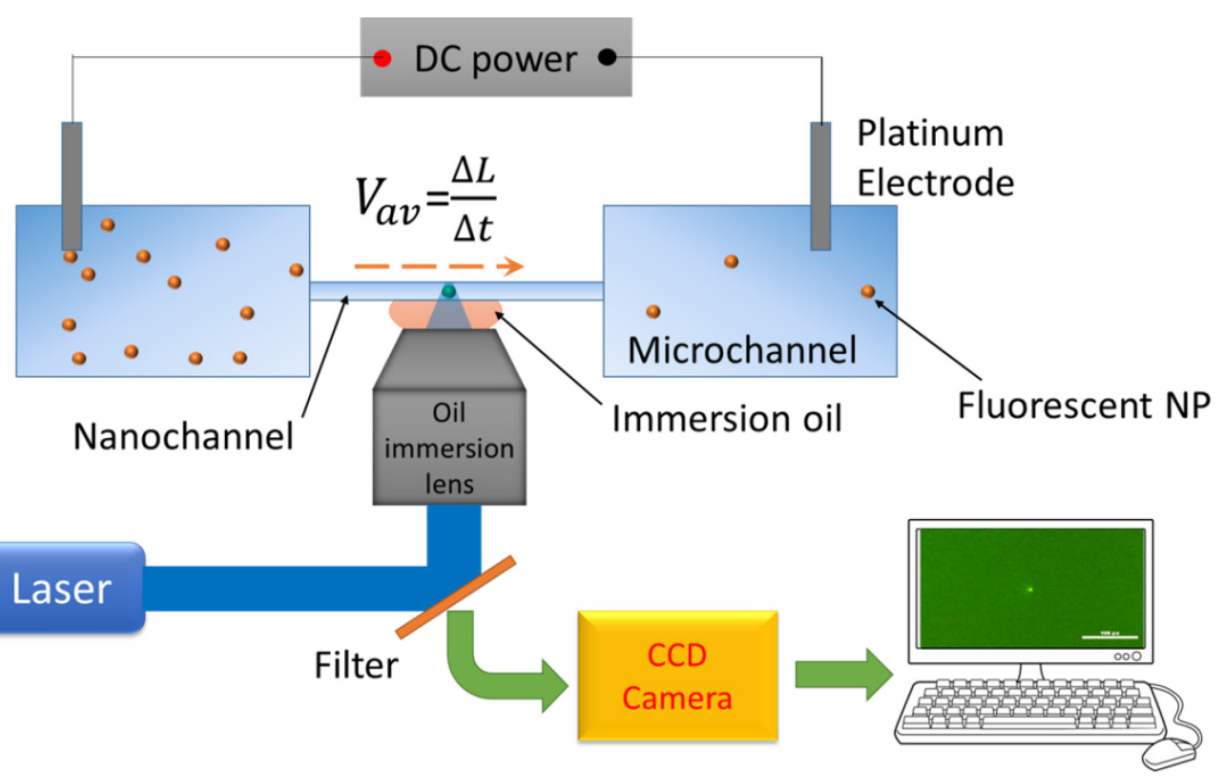

(b)

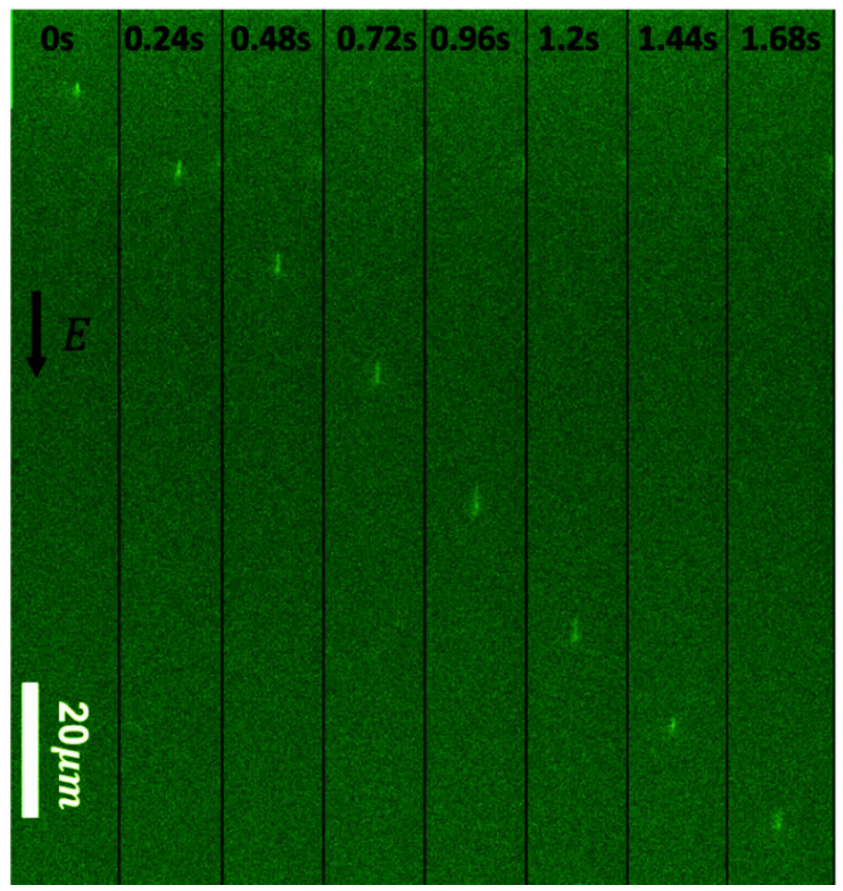

Fig. 2 (a) Schematic of the fluorescent particle tracking system, and (b) an example of electrokinetic motion of a $140 \mathrm{~nm}$ particle in a $340 \mathrm{~nm}$ deep nanochannel under $20 \mathrm{~V} / \mathrm{cm}$ electric field. The video was captured by a CCD camera (Q-imaging, Retiga 2000R) with an exposure time setting of $80 \mathrm{~ms}$.

\subsection{Chemical reagents and experimental setup}


The particle-solution samples were prepared by diluting green carboxylated polystyrene fluorescent nanoparticles (Bangs Laboratories Inc.) into $\mathrm{LiBr}$ solutions. The particle concentration used in the experiments is diluted to about $10^{9} / \mathrm{mL}$. Three sizes of nanoparticles, $60 \mathrm{~nm}, 83 \mathrm{~nm}$ and $140 \mathrm{~nm}$, are used in this study. The ionic concentrations of LiBr solutions used in this work include $10^{-5} \mathrm{M}, 10^{-4} \mathrm{M}, 10^{-3} \mathrm{M}$, and $10^{-2} \mathrm{M}$. The buffer solutions were obtained by dissolving $\mathrm{LiBr}$ (Fisher Scientific) into pure water (Mini Q, Direct-Q3, 18.2 $\mathrm{M} \Omega \cdot \mathrm{cm})$. The length of the nanochannels is $300 \mu \mathrm{m}$. DC electric fields from $1 \mathrm{kV} / \mathrm{m}$ to 10 $\mathrm{kV} / \mathrm{m}$ were applied over the $300 \mu \mathrm{m}$ long nanochannels. To study the particle-to-channel size ratio effects on the electrokinetic motion of nanoparticles, 6 nanochannels and 2 straight microchannels were fabricated. Tab. 1 gives the details of the channels. All the channels are measured by the microscope or the AFM for at least 5 times. Chip conditioning is conducted by loading $\mathrm{LiBr}$ solutions into the fresh channels for 15 minutes before nanoparticle samples are loaded. It should be noted that the buffer solution used for the chip conditioning is the same as that of the nanoparticle sample solutions. For each working condition, i.e. one channel size, one particle size, one ionic concentration and one electric field, the experiments were repeated for at least three times on three independent fresh chips; and for each chip, the motion of the nanoparticles was tracked for at least 10 times. The motion of the nanoparticles was captured by the CCD camera with an exposure time setting of $80 \sim 150 \mathrm{~ms}$. For large nanoparticles, for example, $140 \mathrm{~nm}$ nanoparticles, the fluorescent light intensity is high and the exposure time setting was relatively short, $80 \mathrm{~ms}$; however, for smaller nanoparticles, for example, $60 \mathrm{~nm}$ nanoparticles, the exposure time setting was longer $(150 \mathrm{~ms})$ to make sure the accuracy of the measurement. All the experiments were conducted at room temperature of about $25{ }^{\circ} \mathrm{C}$. For all the cases, only the apparent velocity was measured. The apparent velocity is the combined result of the electrophoresis velocity of the particle and the velocity of the electroosmotic flow inside the channel, and is more useful for the practical applications. 
Tab. 1 Sizes of the PDMS-glass microchannels and the PDMS-glass nanochannels.

\begin{tabular}{|c|c|c|}
\hline Channel No. & Width (w) & Depth (b) \\
\hline 1 & $50 \mu m$ & $25 \mu m$ \\
\hline 2 & $10 \mu \mathrm{m}$ & $5 \mu \mathrm{m}$ \\
\hline 3 & $501 \pm 24 \mathrm{~nm}$ & $340 \pm 17 \mathrm{~nm}$ \\
\hline 4 & $460 \pm 15 \mathrm{~nm}$ & $157 \pm 13.5 \mathrm{~nm}$ \\
\hline 5 & $436 \pm 18 \mathrm{~nm}$ & $102 \pm 5.6 \mathrm{~nm}$ \\
\hline 6 & $596 \pm 11 \mathrm{~nm}$ & $91 \pm 5 \mathrm{~nm}$ \\
\hline 7 & $450 \pm 25 \mathrm{~nm}$ & $83 \pm 6.5 \mathrm{~nm}$ \\
\hline 8 & $507 \pm 11 \mathrm{~nm}$ & $67 \pm 3 \mathrm{~nm}$ \\
\hline
\end{tabular}

\section{Results and discussion}

\subsection{Ionic concentration effects}

Nanoparticles of $60 \mathrm{~nm}$ and $140 \mathrm{~nm}$ in diameter in $10^{-2} \mathrm{M}, 10^{-3} \mathrm{M}, 10^{-4} \mathrm{M}$ and $10^{-5} \mathrm{M}$ $\mathrm{LiBr}$ solutions were tested in two nanochannels with cross-sections of $340 \times 501 \mathrm{~nm}$ and 157 $\times 460 \mathrm{~nm}$, respectively, as well as in a microchannel with a cross-section of $25 \times 50 \mu \mathrm{m}$, with an applied electric field of $2 \mathrm{kV} / \mathrm{m}$. The apparent velocity of the $60 \mathrm{~nm}$ and $140 \mathrm{~nm}$ nanoparticles is showing in Fig. 3, in which $a$ is the diameter of the nanoparticles and $b$ is the depth of the channels. From Fig. 3 one can see that in the microchannel, the apparent velocity of $140 \mathrm{~nm}$ nanoparticles increases with the decreasing ionic concentration from $45 \mu \mathrm{m} / \mathrm{s}$ to $110 \mu \mathrm{m} / \mathrm{s}$, which is consistent with the dependence of the electroomostic flow on the ionic concentration (Peng and Li 2016b). The increase of the electroosmotic flow is due to the increase in zeta potential of the microchannel wall surfaces. However, the zeta potential of the nanoparticles also increases with the decreasing ionic concentration. From the results one can conclude that the influence of the increase in particle zeta poetical is minor and the apparent velocity of the nanoparticles is dominated by the electroosmotic flow in the channel.

In the two nanochannels, the apparent velocity of both $60 \mathrm{~nm}$ and $140 \mathrm{~nm}$ nanoparticles increases as the ionic concentration decreases from $10^{-2} \mathrm{M}$ to $10^{-3} \mathrm{M}$. However, as the ionic concentration decreases further, the velocity of both the $60 \mathrm{~nm}$ and $140 \mathrm{~nm}$ nanoparticles experiences a decreasing trend, which is opposite to that in the microchannel. Obviously, for 
the cases of $10^{-2} \mathrm{M}$ and $10^{-3} \mathrm{M}$ solutions in nanochannels, the thickness of the EDL is very thin, about $10 \mathrm{~nm}$ and $40 \mathrm{~nm}$, respectively; as a result, there is no overlap of EDLs in these two nanochannels, the electroosmotic flow in these two nanochannels is the same as that in the microchannel, increasing with the decrease in ionic concentration, similar to that in the microchannel as mentioned above. By contrast, as the ionic concentration decreases further, to $10^{-4} \mathrm{M}$ and $10^{-5} \mathrm{M}$, the thickness of the EDL adjacent to the channel walls increases dramatically, to about $120 \mathrm{~nm}$ and $400 \mathrm{~nm}$ respectively; as a consequence, EDLs are overlapped in the two nanochannels, resulting in a decreasing electroosmtic flow inside the nanochannels. The effects of overlap of EDLs on the electroomotic flow in nanochannels can be found in the literature (Peng and Li 2016b). In addition, as the ionic concentration decreases, the increase of zeta potential of the polystyrene nanoparticles will also give rise to larger electrophoretic mobility values of the nanoparticles, but in the opposite direction of the electroosmotic flow. As a result, the apparent velocity decreases further, to about $38 \mu \mathrm{m} / \mathrm{s}$ and $55 \mu \mathrm{m} / \mathrm{s}$ for the cases of $60 \mathrm{~nm}$ particles in the $157 \mathrm{~nm}$ deep and $340 \mathrm{~nm}$ deep nanochannels with $10^{-5} \mathrm{M}$ ionic concentration environment. 


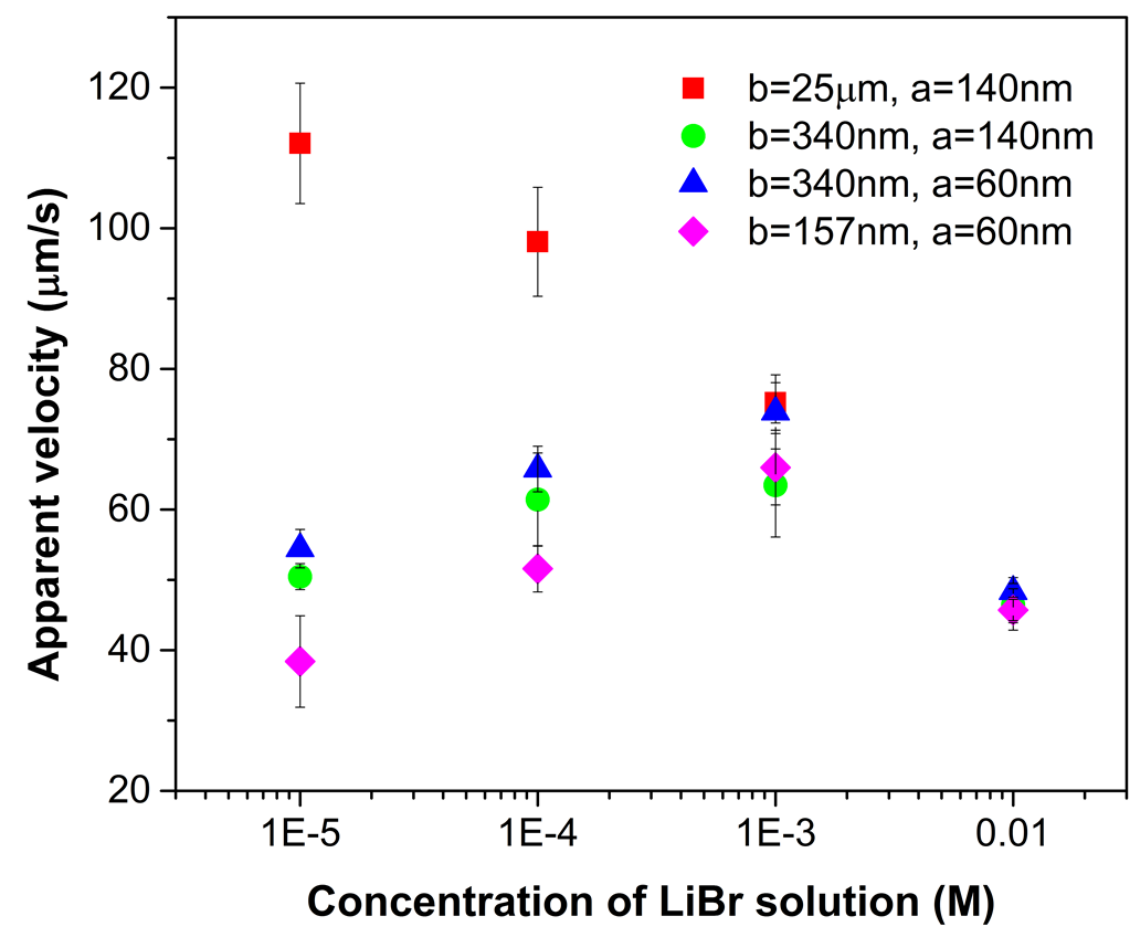

Fig. 3 Concentration effects on the electrokinetic velocity of single nanoparticles in single nanochannels. Apparent velocity of $60 \mathrm{~nm}$ particles and $140 \mathrm{~nm}$ nanoparticles in single nanochannels of $340 \times 501 \mathrm{~nm}$ cross section and, $157 \times 460 \mathrm{~nm}$ cross section, and in a microchannel of $25 \times 50 \mu \mathrm{m}$ was measured by the particle tracking system. For all the cases, the electric field applied over the nanochannels and the microchannel is $2 \mathrm{kV} / \mathrm{m}$ and the ionic concentrations of the $\mathrm{LiBr}$ solution are $10^{-2} \mathrm{M}, 10^{-3} \mathrm{M}, 10^{-4} \mathrm{M}$ and $10^{-5} \mathrm{M}$, and for each data point, the experiments were repeated for at least 30 times. Here, $a$ is the diameter of the nanoparticles, and $b$ is the depth of channels.

Interactions between the EDLs of the nanochannels and the nanoparticles affect the apparent velocity of the nanoparticles. As the ionic concentration decreases, the thickness of EDL near both the nanochannel walls and the nanoparticle surface increases and EDLs may overlap in the gaps between the nanochannel walls and the nanoparticles easily, especially for the cases of large particle-to-channel size ratio systems. For example, in the case of $157 \mathrm{~nm}$ nanochannel 
loaded with $60 \mathrm{~nm}$ nanoparticles in $10^{-3} \mathrm{M} \mathrm{LiBr}$ solution, no overlap of EDL of the opposite nanochannel walls, because the thickness of the EDL is about $40 \mathrm{~nm}$ which is much smaller than the half depth of the nanochannel, about $80 \mathrm{~nm}$. However, if a $60 \mathrm{~nm}$ nanoparticle was loaded in this nanochannel, the gap between the nanoparticle surface and the channel wall should be around $50 \mathrm{~nm}$, assuming that the nanoparticle is located in the middle of the nanochannel due to EDL repulsion (Liu et al. 2014). Consequently, the thickness of the EDL, $40 \mathrm{~nm}$, is larger than the half length of the gap, $25 \mathrm{~nm}$, which means EDLs get overlapped between the nanoparticle and the nanochannel walls. As seen in Fig. 3, the apparent velocity of the $60 \mathrm{~nm}$ nanoparticle is smaller than that in the microchannel. For the cases of particle motion in an overlap EDL, it is hard to find an analytical solution for the nanoparticle transport behavior due to the sophisticated interactions between the particle and the channel wall. However, compared with the cases without overlap of EDL mentioned above, the zeta potential changes are the same, and only the overlap EDL matters. Consequently, we can predict that the electrokinetic motion of nanoparticles in nanochannels filling with diluted ionic solutions is dominated by the overlap EDL and also the overlapping of EDLs between the nanoparticle and the nanochannel walls under the condition of low ionic concentration contributes to a smaller apparent velocity.

\subsection{Effects of particle size to channel size ratio}

Fig. 4 depicts the channel size effects on the electrokinetic motion of $60 \mathrm{~nm}$ nanoparticles in nanochannels of $340 \mathrm{~nm}, 157 \mathrm{~nm}, 102 \mathrm{~nm}, 91 \mathrm{~nm}$ and $84 \mathrm{~nm}$ in depth as well as in microchannels of $25 \mu \mathrm{m}$ and $5 \mu \mathrm{m}$ in depth with $10^{-2} \mathrm{M}$ and $10^{-5} \mathrm{M}$ LiBr solution environment. The applied electric field is $2 \mathrm{kV} / \mathrm{m}$ for all the cases. In Fig. 4, the size ratio $a / b$ is plotted as the $x$-axis, where $a$ is the diameter of the nanoparticle and $b$ is the depth of the channels. It is obvious that the apparent velocity of the nanoparticles decreases with the size ratio for both concentration values. For instance, for the $10^{-2} \mathrm{M}$ cases, the apparent velocity 
of $60 \mathrm{~nm}$ nanoparticles in the microchannels $(a / b \approx 0)$ and large nanochannels (340 $\mathrm{nm}$ deep nanochannel, $a / b=0.18$ ) is about $50 \mu \mathrm{m} / \mathrm{s}$, however, as the channel size decreases to $84 \mathrm{~nm}$ $\operatorname{deep}(a / b=0.71)$, the apparent velocity decreases gradually to about $22 \mu \mathrm{m} / \mathrm{s}$.

Similarly, for the cases of $10^{-5} \mathrm{M}$ ionic concentration, the velocity of $60 \mathrm{~nm}$ nanoparticles in the microchannels is about $110 \mu \mathrm{m} / \mathrm{s}$; however, when the channel size decreases down to nanometer scale $(a / b>0.18$ in Fig. 4), the apparent velocity of the nanoparticles decreases dramatically due to interactions between EDLs, because the thickness of the EDL for the $10^{-5} \mathrm{M}$ case is very large, is about $400 \mathrm{~nm}$, and EDLs are overlapped between nanochannel walls and also between the nanochannel walls and the nanoparticles. When EDLs get overlapped, the electroosmotic flow in the nanochannel will decrease, and the interactions between the nanoparticles and the channel walls become stronger, consequently, the apparent velocity decreases rapidly with the channel size. For example, the velocity of the $60 \mathrm{~nm}$ nanoparticles is about $55 \mu \mathrm{m} / \mathrm{s}$ in the $340 \mathrm{~nm}$ deep nanochannel $(a / b=0.18)$, and this value decreases to about $20 \mu \mathrm{m} / \mathrm{s}$ as the channel size decreases to $91 \mathrm{~nm}(a / b=0.66)$, in which EDLs get overlapped strongly. It can be concluded that, as channel size becomes smaller and the size ratio becomes larger, interactions between the EDLs will dominate the electrokinetic motion of the nanoparticles, resulting in a decrement of the apparent velocity.

It should be noted, under the condition of strong EDL interactions, for example, for the case of $60 \mathrm{~nm}$ nanoparticles in an $84 \mathrm{~nm}$ deep nanochannel $(a / b=0.71)$ filled with $10^{-5} \mathrm{M} \mathrm{LiBr}$ solution, the nanoparticles can hardly move under low electric fields. Aggregation occurs in the nanochannel due to the strong particle-nanochannel electrostatic interactions. An example of $60 \mathrm{~nm}$ nanoparticles trapped in an $84 \mathrm{~nm}$ deep nanochannel is shown in Fig. S1 (a) of the Electronic Supplementary Material. The nanoparticles can only be removed with very high electric field $\left(10^{6} \mathrm{~V} / \mathrm{m}\right.$ or higher). Trapping of DNAs inside nanopores under the condition of 
low electric field has also been reported. (Ai et al. 2010) Attempt of transporting $60 \mathrm{~nm}$ nanoparticles in a $67 \mathrm{~nm}$ deep nanochannel $(a / b \approx 0.9)$ indicates that it is extremely hard to load $60 \mathrm{~nm}$ nanoparticles into such a small nanochannel by electroosmotic flow due to strong DEP (dielectrophoresis) force at the channel entrance (Dongqing Li 2008) or the electrostatic repulsion force between the nanoparticles and the channel wall surfaces. Generally, $60 \mathrm{~nm}$ nanoparticles are stuck at the entrance of the $67 \mathrm{~nm}$ nanochannel, as shown in Fig. S1 (b).

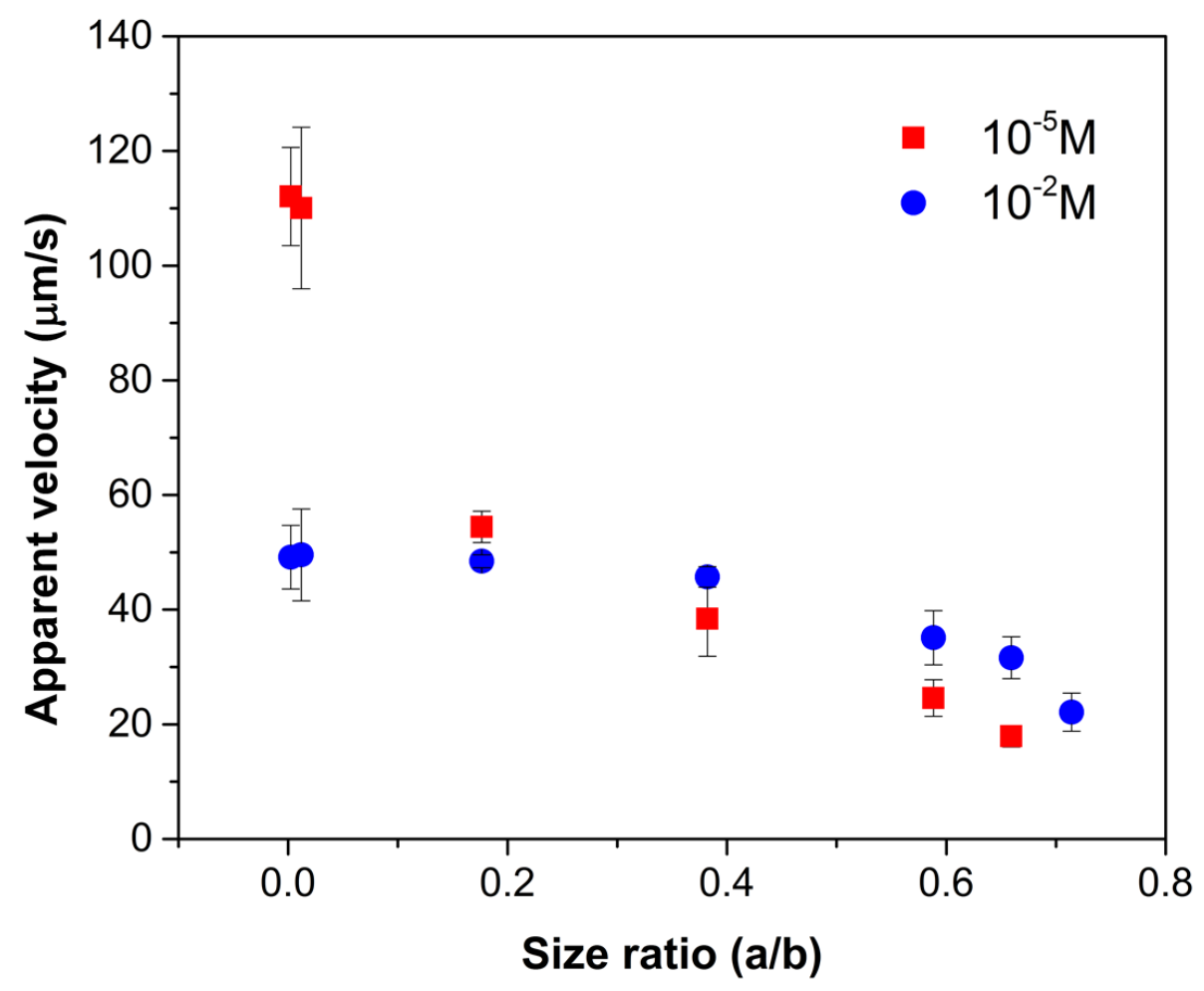

Fig. 4 Particle-to-channel size ratio effects on the apparent velocity of $60 \mathrm{~nm}$ nanoparticle in nanochannels of $340 \mathrm{~nm}, 157 \mathrm{~nm}, 102 \mathrm{~nm}, 91 \mathrm{~nm}$ and $84 \mathrm{~nm}$ in depth as well as in microchannels of $25 \mu \mathrm{m}$ and $5 \mu \mathrm{m}$ in depth with $10^{-2} \mathrm{M}$ and $10^{-5} \mathrm{M} \mathrm{LiBr}$ solution, where $a$ is the diameter of the nanoparticles and $b$ is the depth of the channels. The applied electric field is $2 \mathrm{kV} / \mathrm{m} \mathrm{m}$ for all the cases, and for each case, the experiments were repeated for at least 30 times. 


\subsection{Electric field effects}

Fig. 5 depicts the electric field effects on the apparent velocity of $60 \mathrm{~nm}$ nanoparticles in a $157 \mathrm{~nm}$ deep nanochannel filled with $10^{-2} \mathrm{M}$ buffer solution, $140 \mathrm{~nm}$ particles in a $25 \mu \mathrm{m}$ deep microchannel filled with $10^{-2} \mathrm{M}$ buffer solution, and $140 \mathrm{~nm}$ particles in a $340 \mathrm{~nm}$ deep nanochannel loaded with $10^{-3} \mathrm{M} \mathrm{LiBr}$ solution, respectively. The electric field ranges from 1 $\mathrm{kV} / \mathrm{m}$ to $10 \mathrm{kV} / \mathrm{m}$. It is obvious that the apparent velocity of the nanoparticles increases almost linearly with the applied electric field for all the cases. The slopes of these curves indicate the mobility values of these particles. From Fig. 5 one can see that in $10^{-2} \mathrm{M} \mathrm{LiBr}$ solution, the motion of $60 \mathrm{~nm}$ nanoparticles in the nanochannel of $157 \mathrm{~nm}$ deep is smaller than that of $140 \mathrm{~nm}$ particles in the $25 \mu \mathrm{m}$ deep microchannel due to the interactions of the overlapped EDLs as mentioned above. In addition, electrokinetic motion of $140 \mathrm{~nm}$ nanoparticles in the $25 \mu \mathrm{m}$ deep microchannel filled with $10^{-2} \mathrm{M} \mathrm{LiBr}$ solution is smaller than that in the $340 \mathrm{~nm}$ deep nanochannel filled with $10^{-3} \mathrm{M} \mathrm{LiBr}$ solution. This is because the electroosmotic flow in the channel is much weaker in the high ionic concentration $\left(10^{-2} \mathrm{M}\right)$ solution. In Fig. 5, under the condition of higher electric fields, the error bars of the measured velocity are larger compared with that of the low electric field cases due to the limitation of the fluorescent tracking system. Under the condition of a high electric field, the velocity of the fluorescent particles is high and a sufficient exposure time is needed to clearly observe the nanoparticles. However, a longer exposure time makes the trajectories of the nanoparticles blurring and results in larger errors during the velocity calculation. "Stick/slip" motion of the nanoparticles under the conditions of low electric field and large size ratio has also been observed during the experiments, as shown in Fig. S1 (a). The "stick/slip" motion was previously observed in the experimental studies of translocation of DNAs through nanochannels probably due to strong electrostatic interactions between the negatively charged DNAs and the positively charged channel wall (Uba et al. 2015). However, in this case, both 
the nanochannel wall and the nanoparticles are negatively charged, the "stick/slip" behaviour may due to strong inhomogeneous electric field inside the channel caused by the surface roughness of the nanochannel wall.

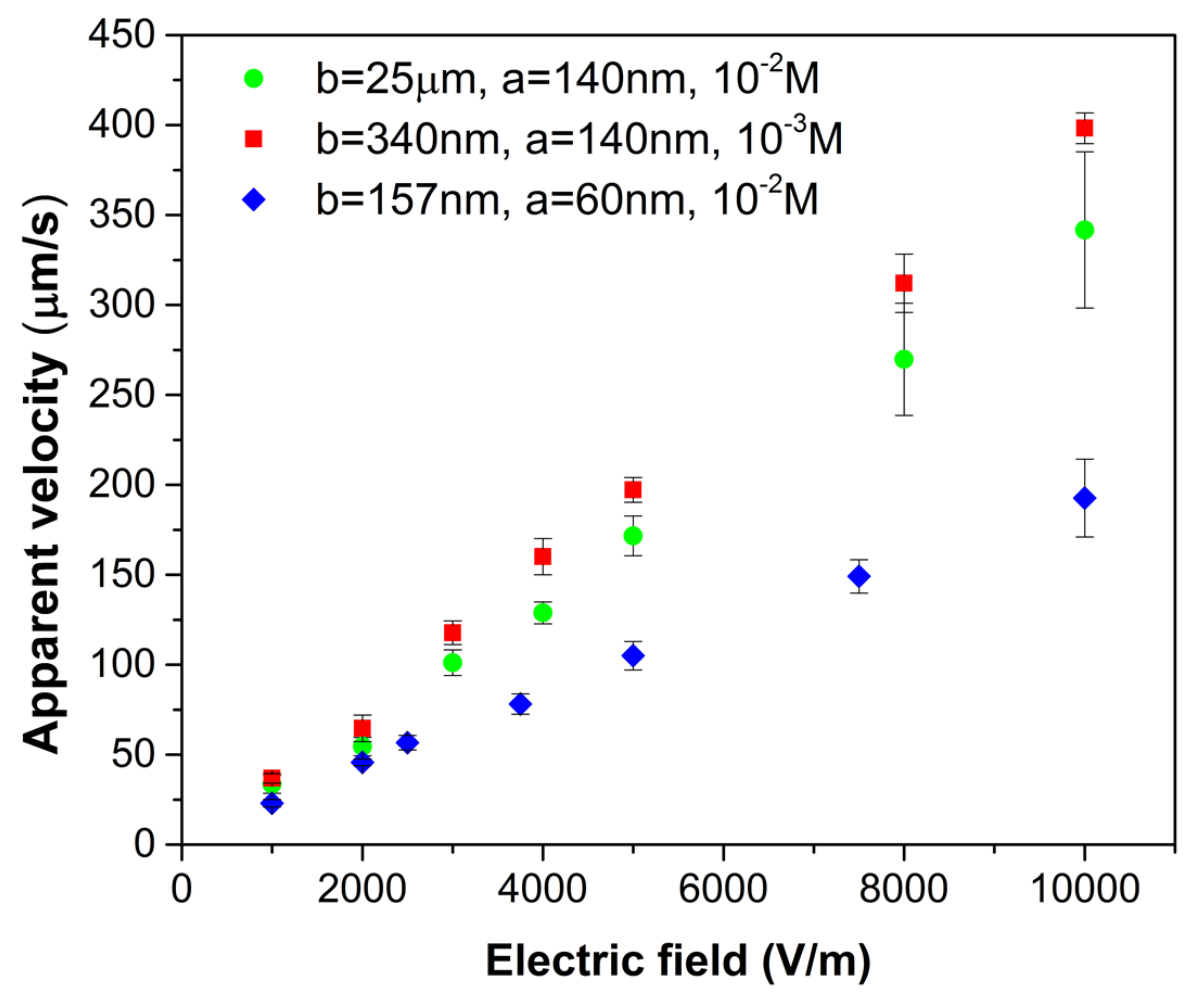

Fig. 5 Electric field effects on the apparent velocity of $60 \mathrm{~nm}$ nanoparticles in a $157 \mathrm{~nm}$ deep nanochannel filled with $10^{-2} \mathrm{M} \mathrm{LiBr}$ solution, and $140 \mathrm{~nm}$ nanoparticles in a $25 \mu \mathrm{m}$ deep microchannel filled with $10^{-2} \mathrm{M}$ LiBr solution and in a $340 \mathrm{~nm}$ deep nanochannel loaded with $10^{-3} \mathrm{M}$ LiBr solution. The applied electric field ranges from $1 \mathrm{kV} / \mathrm{m}$ to $10 \mathrm{kV} / \mathrm{m}$, and for each case, the experiments were repeated for at least 30 times.

\section{Conclusions}

This paper presents a systematical experimental study of electrokinetic motion of single nanoparticles in single nanochannels. PDMS-glass micro-nanochannel chips with single nanochannels are developed. The effects of ionic concentration, particle-to-channel size ratio 
$(\mathrm{a} / \mathrm{b})$, and electric field on the apparent velocity of electrophoretic motion fluorescent nanoparticles in six nanochannels and two microchannels by particle tracking method are investigated. The results show that the electrokinetic velocity of nanoparticles in small single nanochannels increases with the ionic concentration in diluted solutions $\left(<10^{-3} \mathrm{M}\right)$ and decreases with the ionic concentration in concentrated solutions $\left(>10^{-3} \mathrm{M}\right)$. The apparent velocity of single nanoparticles in single nanochannels decreases with increasing particle size to channel size ratio $(a / b)$ due to the interactions of the overlapped EDLs inside the nanochannels. Under the condition of a proper electric field range, the apparent velocity increases with the applied electric field linearly. "Stick-slip" motion and stuck of nanoparticles in single nanochannels under the conditions of large size ratio and low electric field have also been observed. The experimental study presented in this article provides improved understanding of electrokinetic motion of nanoparticles in nanospaces and is potentially valuable for the future applications nanofluidics.

\section{Acknowledgements}

The authors wish to thank the financial support of the Natural Sciences and Engineering Research Council (NSERC) of Canada through a research grant to D. Li.

\section{References}

Ai Y, Liu J, Zhang B, Qian S (2010) Field Effect Regulation of DNA Translocation through a Nanopore. Anal Chem 82:8217-8225.

Chen SB, Keh HJ (1988) Electrophoresis in a dilute dispersion of colloidal spheres. AIChE J 34:1075-1085. doi: 10.1002/aic.690340704

Chen WJ, Keh HJ (2013) Electrophoresis of a charged soft particle in a charged cavity with arbitrary double-layer thickness. J Phys Chem B 117:9757-9767. doi:

10.1021/jp405357e

Davenport M, Healy K, Pevarnik M, et al (2012) The role of pore geometry in single 
nanoparticle detection. ACS Nano 6:8366-8380. doi: 10.1021/nn303126n

Delgado A V., González-Caballero F, Hunter RJ, et al (2007) Measurement and interpretation of electrokinetic phenomena. J Colloid Interface Sci 309:194-224. doi:

$10.1016 /$ j.jcis.2006.12.075

Ding JM, Keh HJ (2001) The Electrophoretic Mobility and Electric Conductivity of a Concentrated Suspension of Colloidal Spheres with Arbitrary Double-Layer Thickness. J Colloid Interface Sci 236:180-193. doi: 10.1006/jcis.2000.7383

Dongqing Li (2008) Encyclopedia of Microfluidics and Nanofluidics.

Duan C, Wang W, Xie Q (2013) Fabrication of nanofluidic devices. Biomicrofluidics 7:26501. doi: 10.1063/1.4794973

Dukhin SS (1993) Non-equilibrium electric surface phenomena. Adv Colloid Interface Sci 44:1-134. doi: 10.1016/0001-8686(93)80021-3

Ennis J, Anderson J (1997) Boundary Effects on Electrophoretic Motion of Spherical Particles for Thick Double Layers and Low Zeta Potential. J Colloid Interface Sci 185:497-514. doi: 10.1006/jcis.1996.4596

Fu J, Schoch RB, Stevens AL, et al (2007) A patterned anisotropic nanofluidic sieving structure for continuous-flow separation of DNA and proteins. Nat Nanotechnol 2:121128.

H. O (1995) Electrophoresis of soft particles. Adv Colloid Interface Sci 62:189. doi: 10.1016/0001-8686(95)00279-Y

Han R, Wang G, Qi S, et al (2012) Electrophoretic migration and axial diffusion of individual nanoparticles in cylindrical nanopores. J Phys Chem C 116:18460-18468. doi: 10.1021/jp303855d

Harms ZD, Haywood DG, Kneller AR, et al (2015) Single-particle electrophoresis in nanochannels. Anal Chem 87:699-705. doi: 10.1021/ac503527d

Henry DC (1931) The cataphoresis of suspended particles. Part I. The equation of cataphoresis. In: Proceedings of the Royal Society of London A: Mathematical, Physical and Engineering Sciences. The Royal Society, pp 106-129

Hsu J-P, Chen Z-S (2007) Electrophoresis of a Sphere along the Axis of a Cylindrical Pore:\&nbsp; Effects of Double-Layer Polarization and Electroosmotic Flow. Langmuir 23:6198-6204.

Hsu JP, Ku MH (2005) Boundary effect on electrophoresis: Finite cylinder in a cylindrical pore. J Colloid Interface Sci 283:592-600. doi: 10.1016/j.jcis.2004.09.004

Hsu JP, Ku MH, Kao CY (2004) Electrophoresis of a spherical particle along the axis of a cylindrical pore: Effect of electroosmotic flow. J Colloid Interface Sci 276:248-254. doi: $10.1016 /$ j.jcis.2004.03.025

Hsu JP, Lo HM, Yeh LH, Tseng S (2012a) Importance of boundary on the electrophoresis of a soft cylindrical particle. J Phys Chem B 116:12626-12632. doi: 10.1021/jp305473s 
Hsu JP, Yee CP, Yeh LH (2012b) Importance of electroosmotic flow and multiple ionic species on the electrophoresis of a rigid sphere in a charge-regulated zwitterionic cylindrical Pore. Langmuir 28:10942-10947. doi: 10.1021/la3018634

Huang C-H, Hsu H-P, Lee E (2012) Electrophoretic motion of a charged porous sphere within micro- and nanochannels. Phys Chem Chem Phys 14:657-667. doi: 10.1039/C1CP21938C

Hulings ZK, Melnikov D V., Gracheva ME (2015) Charged nanoparticle in a nanochannel: Competition between electrostatic and dielectrophoretic forces. Phys Rev E - Stat Nonlinear, Soft Matter Phys 91:1-7. doi: 10.1103/PhysRevE.91.062713

Jeffet J, Kobo A, Su T, et al (2016) Super-Resolution Genome Mapping in Silicon Nanochannels. ACS Nano acsnano.6b05398. doi: 10.1021/acsnano.6b05398

Keh H, Anderson J (1985) Boundary Effects on Electrophoretic Motion of Colloidal Spheres. J Fluid Mech 153:417-439.

Keh H, Chen S (1988) Electrophoresis of a Colloidal Sphere Parallel To a Dielectric Plane. J Fluid Mech 194:377-390.

Keh HJ, Chen JB (1993) Particle Interactions in Electrophoresis .5. Motion of Multiple Spheres with Thin but Finite Electrical Double-Layers. J Colloid Interface Sci 158:199_ 222. doi: $10.1006 /$ jcis. 1993.1248

Keh HJ, Chiou JY (1996) Electrophoresis of a colloidal sphere in a circular cylindrical pore. AIChE J 42:1397-1406. doi: 10.1002/aic.690420520

Keh HJ, Hsieh TH (2008) Electrophoresis of a colloidal sphere in a spherical cavity with arbitrary zeta potential distributions and arbitrary double-layer thickness. Langmuir 24:390-398. doi: 10.1021/la702399u

Keh HJ, Yang FR (1991) Particle Interactions in Electrophoresis .4. Motion of Arbitrary 3Dimensional Clusters of Spheres. J Colloid Interface Sci 145:362-389.

Lee TC, Keh HJ (2014) Electrophoresis of a spherical particle in a spherical cavity. Microfluid Nanofluidics 16:1107-1115. doi: 10.1007/s10404-013-1276-8

Li D (2004) Electrokinetics in microfluidics. Academic Press

Li D, Daghighi Y (2010) Eccentric electrophoretic motion of a rectangular particle in a rectangular microchannel. J Colloid Interface Sci 342:638-642. doi: 10.1016/j.jcis.2009.10.047

Li WC, Keh HJ (2016) Electrophoretic mobility of charged porous shells or microcapsules and electric conductivity of their dilute suspensions. Colloids Surfaces A Physicochem Eng Asp 497:154-166. doi: 10.1016/j.colsurfa.2016.02.028

Liu YW, Pennathur S, Meinhart CD (2016) Electrophoretic mobility of spherical particles in bounded domain. J Colloid Interface Sci 461:32-38. doi: 10.1016/j.jcis.2015.08.039

Liu YW, Pennathur S, Meinhart CD (2014) Electrophoretic mobility of a spherical nanoparticle in a nanochannel. Phys Fluids. doi: 10.1063/1.4901330 
Loewenberg M, Davis RH (1995) Near-contact electrophoretic particle motion. J Fluid Mech 288:103. doi: 10.1017/S002211209500108X

Masliyah JH, Bhattacharjee S (2006) Electrokinetic and colloid transport phenomena. John Wiley \& Sons

Menard LD, Ramsey JM (2013) Electrokinetically-Driven Transport of DNA through Focused Ion Beam Milled Nano fluidic Channels. Anal Chem 85:1146-1153.

Mijatovic D, Eijkel JCT, van den Berg A (2005) Technologies for nanofluidic systems: topdown vs. bottom-up--a review. Lab Chip 5:492-500. doi: 10.1039/b416951d

Miloh T, Boymelgreen A (2014) Travelling wave dipolophoresis of ideally polarizable nanoparticles with overlapping electric double layers in cylindrical pores. Phys Fluids. doi: $10.1063 / 1.4884956$

Movahed S, Li D (2012) Electrokinetic motion of a rectangular nanoparticle in a nanochannel. J Nanoparticle Res 14:1-15. doi: 10.1007/s11051-012-1032-0

Napoli M, Atzberger P, Pennathur S (2011) Experimental study of the separation behavior of nanoparticles in micro- and nanochannels. Microfluid Nanofluidics 10:69-80. doi: 10.1007/s10404-010-0647-7

Napoli M, Eijkel JCT, Pennathur S (2010) Nanofluidic technology for biomolecule applications: a critical review. Lab Chip 10:957-985. doi: 10.1039/b917759k

Nedelcu S, Sommer JU (2014) Charge inversion effects in electrophoresis of polyelectrolytes in the presence of multivalent counterions and transversal electric fields. Polymers (Basel) 6:2942-2960. doi: 10.3390/polym6122942

O'Brien RW, Hunter RJ (1981) The electrophoretic mobility of large colloidal particles. Can J Chem 59:1878-1887. doi: 10.1139/v81-280

O'Brien RW, White LR (1978) Electrophoretic mobility of a spherical colloidal particle. J Chem Soc Faraday Trans 2 Mol Chem Phys 74:1607-1626.

Ohshima H (1994) A Simple Expression for Henry's Function for the Retardation Effect in Electrophoresis of Spherical Colloidal Particles. J. Colloid Interface Sci. 168:269-271.

Ohshima H (1996) Henry's Function for Electrophoresis of a Cylindrical Colloidal Particle. J Colloid Interface Sci 180:299-301. doi: 10.1006/jcis.1996.0305

Ohshima H (1995) Electrophoretic mobility of soft particles. Colloids Surfaces A Physicochem Eng Asp 103:249-255. doi: 10.1016/0927-7757(95)03293-M

Ohshima H, Healy TW, White LR (1983) Approximate analytic expressions for the electrophoretic mobility of spherical colloidal particles and the conductivity of their dilute suspensions. J Chem Soc Faraday Trans 2 Mol Chem Phys 79:1613-1628.

Peng R, Li D (2016a) Fabrication of polydimethylsiloxane (PDMS) nanofluidic chips with controllable channel size and spacing. Lab Chip 16:3767-3776. doi: 10.1039/C6LC00867D

Peng R, Li D (2015) Fabrication of nanochannels on polystyrene surface. Biomicrofluidics 
Peng R, Li D (2016b) Electroosmotic flow in single PDMS nanochannels. Nanoscale 1223712246. doi: 10.1039/C6NR02937J

Plesa C, Verschueren D, Pud S, et al (2016) Direct observation of DNA knots using a solidstate nanopore. Nat Nanotechnol 1-6. doi: 10.1038/nnano.2016.153

Qian S, Joo SW, Hou W, Zhao X (2008) Electrophoretic Motion of a Spherical Particle with a Symmetric Nonuniform Surface Charge Distribution in a Nanotube. Langmuir 53325340.

Sackmann EK, Fulton AL, Beebe DJ (2014) The present and future role of microfluidics in biomedical research. Nature 507:181-9. doi: 10.1038/nature13118

Schnitzer O, Frankel I, Yariv E (2014) Electrophoresis of bubbles. J Fluid Mech 753:49-79. doi: $10.1017 / \mathrm{jfm} .2014 .350$

Semenov I, Raafatnia S, Sega M, et al (2013) Electrophoretic mobility and charge inversion of a colloidal particle studied by single-colloid electrophoresis and molecular dynamics simulations. Phys Rev E - Stat Nonlinear, Soft Matter Phys 87:1-7. doi:

10.1103/PhysRevE.87.022302

Shugai AA, Carnie SL, Chan DYC, Anderson JL (1997) Electrophoretic Motion of Two Spherical Particles with Thick Double Layers. J Colloid Interface Sci 371:357-371.

Shugai A, Carnie S (1999) Electrophoretic Motion of a Spherical Particle with a Thick Double Layer in Bounded Flows. J Colloid Interface Sci 213:298-315. doi: 10.1006/jcis.1999.6143

Swan JW, Furst EM (2012) A simpler expression for Henry's function describing the electrophoretic mobility of spherical colloids. J Colloid Interface Sci 388:92-94. doi: 10.1016/j.jcis.2012.08.026

Tseng S, Lin C, Hsu J, Yeh L (2013) Electrophoresis of Deformable Polyelectrolytes in a Nanofluidic Channel. Langmuir 29:2446-2454.

Uba FI, Pullagurla SR, Sirasunthorn N, et al (2015) Surface charge, electroosmotic flow and DNA extension in chemically modified thermoplastic nanoslits and nanochannels. Analyst 140:113-26. doi: 10.1039/c4an01439a

Unlu M, Morgan ME, Minden JS (1997) Difference gel electrophoresis: a single gel method for detecting changes in protein extracts. Electrophoresis 18:2071-2077. doi: 10.1002/elps.1150181133

Wang LJ, Keh HJ (2009) Electrophoresis of a cylindrical particle with a nonuniform zeta potential distribution parallel to a charged plane wall. J Phys Chem C 113:12790-12798. doi: $10.1021 /$ jp903077e

Wang N, Yee CP, Chen YY, et al (2013) Electrophoresis of a pH-regulated zwitterionic nanoparticle in a pH-regulated zwitterionic capillary. Langmuir 29:7162-7169. doi: 10.1021/1a400946s

Wang YC, Stevens AL, Han J (2005) Million-fold preconcentration of proteins and peptides 
by nanofluidic filter. Anal Chem 77:4293-4299. doi: 10.1021/ac050321z

Weerakoon-Ratnayake KM, Uba FI, Oliver-Calixte NJ, Soper SA (2016) Electrophoretic Separation of Single Particles Using Nanoscale Thermoplastic Columns. Anal Chem 88:3569-3577. doi: 10.1021/acs.analchem.5b04065

Wiersema P., Loeb a. ., Overbeek JT. (1966) Calculation of the electrophoretic mobility of a spherical colloid particle. J Colloid Interface Sci 22:78-99. doi: 10.1016/00219797(66)90069-5

Xia D, Yan J, Hou S (2012) Fabrication of nanofluidic biochips with nanochannels for applications in DNA analysis. Small 8:2787-801. doi: 10.1002/smll.201200240

Xuan X, Raghibizadeh S, Li D (2006) Wall effects on electrophoretic motion of spherical polystyrene particles in a rectangular poly(dimethylsiloxane) microchannel. J Colloid Interface Sci 296:743-748. doi: 10.1016/j.jcis.2005.09.039

Xuan X, Ye C, Li D (2005) Near-wall electrophoretic motion of spherical particles in cylindrical capillaries. J Colloid Interface Sci 289:286-290. doi: 10.1016/j.jcis.2005.03.045

Yasui T, Rahong S, Motoyama K, et al (2013) DNA Manipulation and Separation in Sublithographic-Scale Nanowire Array. ACS Nano 7:3029-3035.

Yoon BJ, Kim S (1989) Electrophoresis of spheroidal particles. J Colloid Interface Sci 128:275-288. doi: 10.1016/0021-9797(89)90405-0

Zhang X, Hsu WL, Hsu JP, Tseng S (2009) Diffusiophoresis of a soft spherical particle in a spherical cavity. J Phys Chem B 113:8646-8656. doi: 10.1021/jp9014417

Zhou K, Perry JM, Jacobson SC (2011) Transport and Sensing in Nanofluidic Devices. Annu Rev Anal Chem 4:321-341. doi: 10.1146/annurev-anchem-061010-113938 